\title{
El $\underline{v}$ (verbo ligero) como categoría procedimental
}

\author{
Daniel Romero* \\ Universidad Nacional de La Plata, Argentina
}

\begin{abstract}
Resumen
En los trabajos de Leonetti $(2001,2004)$ y Escandell Vidal y Leonetti (1997) se sostiene la hipótesis de que las Categorías Funcionales (CCFF) propuestas en el Programa Minimalista de Chomsky (1995, 1998, 2005) son las categorías procedimentales que propone la Teoría de Relevancia (Sperber y Wilson [1986] 1995). Esto se demuestra convincentemente respecto a algunas de las CCFF, el Determinante y el Complementante, por ejemplo, pero no se menciona a la $\mathrm{CF}$ verbo ligero ( $\underline{\mathrm{v}})$. Este trabajo intentará mostrar que $\underline{\mathrm{v}}$ puede considerarse también como categoría procedimental.
\end{abstract}

Palabras clave: categoría procedimental, categoría funcional, verbo liviano, papel temático, inferencia.

*Para correspondencia, dirigirse a: Daniel Romero (daniel.dromakd@gmail.com), Crámer 381, Piso 12, Departamento 9, CA de Buenos Aires, CP 1426, República Argentina. 


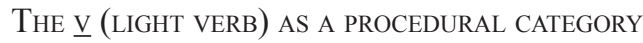

\begin{abstract}
The papers of Leonetti $(2001,2004)$ and Escandell Vidal and Leonetti (1997) hypothesized that the functional categories (FFCC) proposed in the Minimalist Program of Chomsky $(1995,1998,1999,2005)$ are the procedural categories proposed by Relevance Theory (Sperber and Wilson, [1986] 1995). This is demonstrated convincingly for some of the FFCC, the Determinant and the Complementizer, for example, but there is no mention of the FC light verb ( $\underline{v})$. This paper will attempt to show that $\underline{\mathrm{v}}$ can also be considered a procedural category.
\end{abstract}

Key words: procedural category, functional category, light verb, thematic role, infrerence.

Recibido: 08/06/10. Aceptado: 09/09/10.

\title{
1. LAS CATEGORÍAS FUNCIONALES
}

Durante el período que podría llamarse de transición entre el modelo de Principios y Parámetros (Chomsky 1981) y el Programa Minimalista (PM) (Chomsky, 1995, 1998 y 2005) surgió la noción de Categoría Funcional (CF). Las primeras que se propusieron fueron el Complementante (C), como posición en la que se podían insertar constituyentes desplazados, como los SQU- y verbos en las oraciones interrogativas y constituyentes focalizados, y la flexión verbal (FLEX); esta última debido a que desde los primeros modelos de la Gramática Generativa se había observado que los morfemas flexivos del verbo contienen rasgos gramaticales y semánticos que inciden en las estructuras sintácticas. La investigación subsiguiente llevó a proponer que el léxico de una lengua contiene dos clases de elementos: las Categorías Sustantivas (las clases de palabras Nombre (N), Verbo (V), Adjetivo (A) y Preposición (P)) y las Categorías Funcionales (CF), definidas como complejos o haces de rasgos, formales y semánticos. En Chomsky (1998), se señala que:

Los elementos léxicos entran en dos categorías principales: sustantiva y funcional; en este punto nos preocupan principalmente las últimas. Tomemos las Categorías Funcionales Centrales (CCFFCC) como C 
(expresa Fuerza / Modo), T (tiempo, estructura eventual), y v , el núcleo verbo ligero de las construcciones transitivas. Todas las CCFFCC pueden tener rasgos- $\varphi$ (obligatorios para $\mathrm{T}$ y $\underline{\mathrm{v}}$ ). Estos son no-interpretables, constituyendo el centro de los sistemas de concordancia-de Caso (estructural) y movimiento (Mueva) [traducción nuestra].

También se considera una CF el Determinante (D) que, de acuerdo con la hipótesis de Abney (1987), expresa referencialidad.

Los rasgos formales (rasgos- $\varphi$ ) de estas categorías también pueden estar presentes en las Categorías Sustantivas, están representados en los morfemas flexivos, son estrictamente gramaticales (entre estos, se cuentan número, género, persona) y deben cotejarse y descargarse debido a que no siempre tienen interpretación semántica. Los rasgos semánticos de las CCFF se interpretan en Forma Lógica (FL); por ejemplo, la referencialidad que aporta el Determinante (D), la modalidad oracional del Complementante (C) y, obviamente, el Tiempo. $\underline{v}$ posee el rasgo semántico de "transitividad" o "agentividad". Estos rasgos se interpretan en la interfaz FL.

\section{LAS CATEGORÍAS PROCEDIMENTALES}

Por otra parte, la Teoría de la Relevancia (Sperber y Wilson 1986/1995) es una teoría pragmática, pero no toma como objeto solamente el uso del lenguaje en contexto, sino mecanismos de procesamiento inferencial que hacen posible la interpretación de enunciados. Un concepto central de la teoría es el de "comunicación inferencial". La comunicación se considera exitosa cuando el emisor proporciona evidencia de su intención de transmitir una proposición y el oyente infiere su intención de la evidencia aportada por el enunciado. La relevancia no se considera en la acepción de Grice (1975), sino como una propiedad de los enunciados, que puede medirse gradualmente. Un enunciado es más relevante cuánto menos costo cognitivo requiere asignarle una interpretación. Costo cognitivo, una noción cercana a la de Chomsky (1995), se refiere a "esfuerzo de procesamiento", la cantidad de inferencias que deben extraerse del contenido semántico para lograr una interpretación completa del enunciado. La relevancia es un factor más en el proceso cognitivo de interpretación; no es un concepto solamente discursivo, y no siempre depende de la cantidad de información explícita: un enunciado demasiado informativo puede requerir un mayor costo de procesamiento y volverse entonces menos relevante. 
Se toman en cuenta dos clases de contenidos semánticos: los contenidos conceptuales y los contenidos procedimentales (Sperber y Wilson 1986/1995 y Leonetti y Escandell Vidal 1997). Las unidades lingüísticas se distinguen porque su distinto contenido: los morfemas raíces que forman las palabras como sustantivos, adjetivos y verbos codifican conceptos. Por otra parte, “...los marcadores del discurso, las marcas de modalidad oracional, las partículas citativas y evidenciales, la entonación, los tiempos y modos verbales, los determinantes y pronombres definidos, los adverbios deícticos y focalizadores, y los mecanismos sintácticos que determinan la estructura informativa (por ejemplo, los que rigen la asignación del foco)" (Escandell Vidal y Leonetti 1997).

Leonetti (2001) sostiene que las CCFF de la Gramática Generativa son elementos lingüísticos con contenido exclusivamente procedimental, y propone algunas equivalencias: las marcas de modalidad oracional, los tiempos y modos verbales, los determinantes y pronombres definidos son las CCFF del PM, C, T y D, respectivamente.

¿Cómo afectan las categorías procedimentales a la interpretación de un enunciado? Los procesos inferenciales no se limitan solamente a la extracción de implicaturas en el sentido de Grice (1975), a saber, inferencias inductivas extraídas de la relación entre el enunciado y elementos del contexto. Las categorías procedimentales son instrucciones de procesamiento que desencadenan un proceso inferencial que lleva a una representación proposicional completa. Estas representaciones se denominan explicaturas y se originan en dos niveles:

a) Explicaturas proposicionales: consisten en la construcción de la forma proposicional; en este nivel, las categorías procedimentales desambiguan, estableciendo los referentes y el tiempo en que debe ser interpretado el evento expresado.

b) Explicaturas de nivel superior: caracterizan el enunciado como expresión de una intención, como aseverar, preguntar u ordenar. Explicitan la fuerza ilocutoria o modalidad de enunciación.

La interpretación completa del enunciado supone un nivel más, el de las implicaturas; en este nivel, actúan como instrucciones los conectores y marcadores de discurso, que restringen la relación de los distintos contenidos entre sí y con el contexto.

Las categorías procedimentales, como ya se ha dicho, contribuyen en el proceso de inferencia de las explicaturas. Carston (2000) sostiene que las explicaturas constituyen lo comunicado explícitamente por el enunciado, aunque parte de su contenido se infiere por procesos pragmáticos. Una 
explicatura se basa en la FL de una oración, la FL se "enriquece" y el valor veritativo de un enunciado se establece teniendo en cuenta sus explicaturas, no solo su FL:

(1) Llovió mucho; no se puede jugar el partido.

Consideremos el siguiente contexto situacional: un árbitro emite (1) en una entrevista por televisión luego de suspender la realización de un partido de fútbol. El enunciado (1) es argumentativo: la primera oración, llovió mucho, es un argumento o justificación que, si es evaluado como verdadero, legitima tanto el que no se pueda jugar el partido como la suspensión del mismo.

Las condiciones veritativas de llovió mucho se establecen sobre dos clases de material semántico: una parte está codificada por elementos lingüísticos y otra es inferida pragmáticamente. La FL enriquecida o explicatura de llovió mucho es:

(2) El hablante $\boldsymbol{h}$ asevera que existe lluvia en cantidad $\boldsymbol{x}, \boldsymbol{x}$ es superior a la normalidad, en el tiempo $\boldsymbol{t}, \boldsymbol{t}$ es anterior al tiempo en que se emite el enunciado.

Asevera representa la modalidad de enunciación o fuerza ilocutoria, $\boldsymbol{x}$ y $\boldsymbol{t}$ son variables que deben saturarse para que se pueda determinar si el contenido del enunciado es verdadero o falso. Tanto la fuerza ilocutoria como la saturación de las variables surgen de procesos inferenciales activados por la presencia de categorías procedimentales.

\section{LA HIPÓTESIS DE $\underline{\mathrm{V}}$}

Si bien en todos los trabajos ya citados (también en otros de Leonetti) se encuentran análisis que demuestran que las CCFF tienen contenido procedimental, no se menciona $\underline{\mathrm{v}}$, y el objetivo de este trabajo es intentar demostrar que es también una categoría procedimental y que da lugar a explicaturas proposicionales.

La hipótesis de $\underline{\text { se }}$ se propuso en Arad (1998), reformulando la hipótesis de Pollock (1989) que presenta pruebas concluyentes para analizar los morfemas de Tiempo y de Concordancia como proyecciones independientes en el nivel de la representación abstracta. Se conoce con el nombre de Hipótesis de la 
Flexión Escindida (Split-IP Hypothesis). Arad (1998) la modifica teniendo en cuenta que en Chomsky (1995) se propone una reducción del aparato descriptivo, lo que lleva a eliminar las CCFF que no presentan rasgos semánticos, y la propuesta de Hale y Keyser (1993) acerca de la existencia de distintas "capas verbales" (verbal shells) y de que la asignación de papeles temáticos a los argumentos de un predicado surge de la configuración estructural, no de propiedades semánticas intrínsecas del verbo.

Estas cuestiones pueden ejemplificarse en las oraciones:

(3) María tuvo una biblioteca muy completa hasta su divorcio.

(4) María pesó la fruta con una balanza.

De acuerdo con estas dos oraciones, tener asigna Locativo al argumento externo (tradicionalmente sujeto) y pesar Agente. Pero en las siguientes oraciones:

(5) María tuvo un bebé precioso.

(6) María pesó $3,8 \mathrm{~kg}$ al nacer.

vemos que los papeles temáticos que reciben los argumentos externos no son los mismos, a pesar de tratarse del mismo verbo; en (5) es Origen y en (6) Experimentante. La conclusión que surge del trabajo de Hale y Keyser (1993) tiene dos consecuencias:

Primero, que el papel temático del argumento externo resulta de la combinación de propiedades semánticas de la predicación completa, es decir de la relación que establece el V con su complemento.

Segundo, que se deben postular categorías funcionales que puedan cotejar los rasgos de caso no interpretables de los argumentos que relacionan el V, tanto los internos o complementos como el externo, con el sujeto.

Hale y Keyser (1993) propusieron la siguiente estructura para todos los predicados ${ }^{1}$ :

1 Se sustituye SN por SD asumiendo que los argumentos deben tener propiedades referenciales. 
(7)

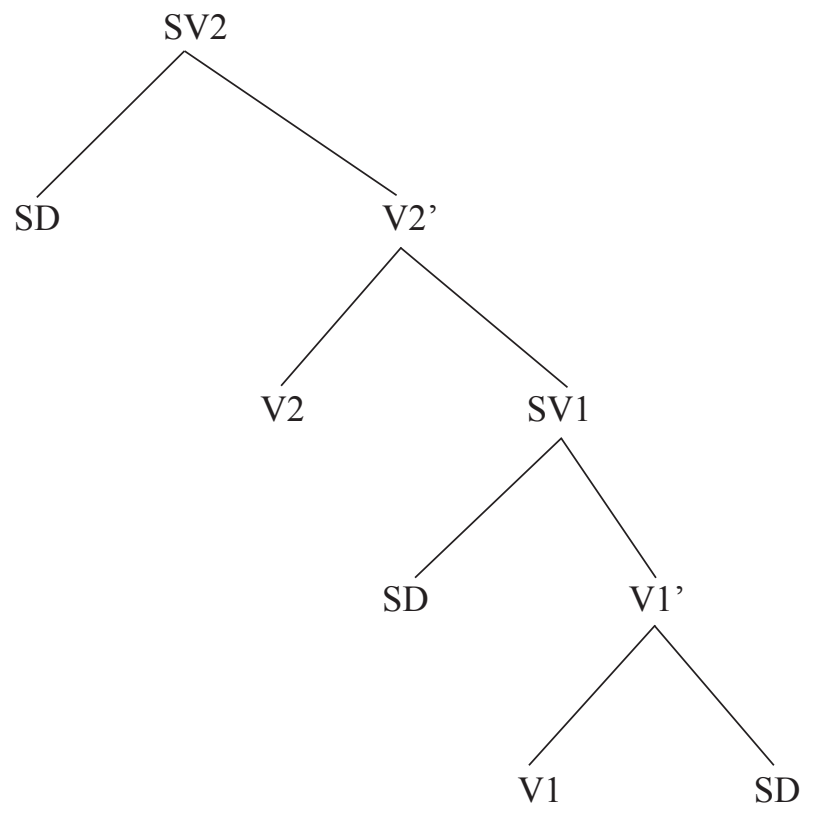

Chomsky (1998) propone la siguiente representación para los predicados transitivos:

(8)

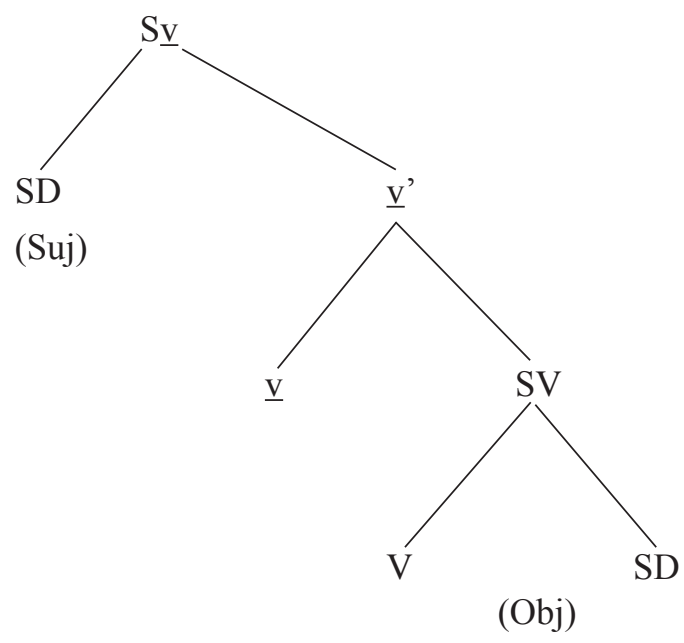




\section{VCOMO CATEGORÍA PROCEDIMENTAL}

La representación de la estructura sintáctica de una oración de Chomsky (1998) permite conservar los logros teóricos obtenidos por la hipótesis de Hale y Keyser (1993). La CF v legitima el agente mediante la asignación estructural de papel temático y coteja el caso acusativo del Obj. Con los rasgos de caso de Suj y Obj cotejados, la derivación converge y alcanza la Materialización.

Los rasgos de caso se expresan en algunas lenguas en la flexión en $\mathrm{NN}$ y AA. En otras, como el español, en algunas estructuras se marcan mediante preposiciones; por ejemplo, si el Obj es un SD que expresa una entidad con los rasgos [+animado] y [+definido], se inserta $a$.

(9) Juan vio $a$ Pedro.

Sin embargo, la inserción de $a$ no se da siempre, depende de los rasgos del SD; podemos comparar:

(10) Una rata vio un gato.

(11) Un gato vio una rata.

Tanto Suj como Obj son SSDD [+animado], pero [-definido]. No nos ocuparemos de cómo interactúan estos dos rasgos y otros en la estructura interna de los SSDD, sino que buscaremos establecer las razones por las que en (10) y (11) se interpreta regularmente que el SD que precede al verbo recibe papel temático de Experimentante y el que lo sigue de Tema, independientemente de sus propiedades semánticas.

(12) Una mujer busca un hombre.

(13) Un hombre busca una mujer.

En (12) y (13) vemos algo similar, el SD que precede al verbo es Agente $\mathrm{y}$ el que lo sigue, Tema.

Asumimos, como se dijo más arriba, que las categorías procedimentales desencadenan un proceso inferencial que condiciona la interpretación de las categorías conceptuales; no modifican el significado conceptual de los elementos léxicos, sino que establecen en qué relación se deben interpretar. Son elementos de interfaz, permiten relacionar diferencias formales (sintácticas y morfológicas) con aspectos del significado. En los casos en que tienen realización léxica, ya sea como palabras o morfemas, esto es bastante 
claro; en algunas lenguas solo una expresión referencial puede aparecer en la función sujeto, como en español:

\section{(14) *Hombre muy alto llegó cansado.}

La ausencia de $\mathrm{D}$ en (14) produce la no convergencia de la derivación y en consecuencia una interpretación defectuosa. En este sentido, también el orden de los constituyentes de una oración afecta a la interpretación; algunas estructuras, aunque aceptables, tienen una interpretación "costosa", porque ofrecen un grado bajo de relevancia, como en:

\section{(15) Al gato temen las ratas.}

Asumimos que los papeles temáticos de los argumentos de un predicado son elementos esenciales para la interpretación, y que forman parte del contenido explícito o significado literal de un enunciado, dado que contribuyen a la asignación de condiciones veritativas. Con factores contextuales idénticos, si (16) es verdadera (17) es necesariamente falsa.

(16) El gato persigue a la rata.

(17) La rata persigue al gato.

Si consideramos correcta la hipótesis de Hale y Keyser (1993), los papeles temáticos se asignan en una configuración jerárquica como la representada en (8), cuyo núcleo es … Esta configuración se proyecta en la estructura del enunciado, que es lineal, no jerárquica. De acuerdo con el Axioma de Correspondencia Lineal de Kayne (1994):

(18) Una categoría a precede a una categoría b si y solo si a manda-c asimétricamente $\mathrm{a} b$.

Las relaciones lineales en el enunciado dependen, entonces, de las relaciones jerárquicas que se establecen en $\mathrm{S} \underline{\mathrm{v}}$; el argumento externo manda$\mathrm{c}$ al argumento interno y, luego de la linealización, lo precede (Suj precede a Obj). Así resulta que las interpretaciones de los papeles temáticos en los ejemplos que repetimos:

(10) Una rata vio un gato.

(11) Un gato vio una rata.

(12) Una mujer busca un hombre.

(13) Un hombre busca una mujer, 
surgen de que se ha linealizado la estructura de Sv y de que en la estructura (lineal) del enunciado se preservan, aunque modificadas, las relaciones jerárquicas establecidas por el núcleo $\underline{\underline{ }} .^{2}$

Además, la estructura lineal se relaciona con la distribución de la información en el enunciado. La posición preverbal del SUJ se considera en español y muchas otras lenguas como temática o de información conocida. Con predicados monádicos u oraciones pasivas que admiten SUJ postverbal se considera topicalizado al SUJ cuando aparece antes del verbo, como puede verse en los ejemplos que siguen:

(14) Escapó un preso. / UN PRESO escapó.

(15) Quedaron algunas tareas inconclusas. / ALGUNAS TAREAS quedaron inconclusas.

(16) Se revisaron todos los datos. / TODOS LOS DATOS se revisaron.

Por esto también puede sostenerse que el $\underline{v}$ también contiene instrucciones que permiten interpretar la estructura informativa del enunciado, consistentes en marcar la diferencia entre tema y rema.

Es en este sentido que creemos que se puede sostener que $\underline{v}$ es también una categoría procedimental, dado que permite inferir las relaciones semánticas que establecen las entidades referenciales, independientemente de cuál sea su contenido y que afecta en cierta medida a la estructura informativa.

\section{REFERENCIAS BIBLIOGRÁFICAS}

Abney, S. P. 1987. The English Noun Phrase and its Sentential Aspect. Ph.D. dissertation, MIT.

Ahern, Aoife y Manuel Leonetti. 2004. Procedural semantics and pragmatic inference. En Rosina Márquez-Reiter y María Elena Placencia (eds.). Current Trends in the Pragmatics of Spanish. Amsterdam: John Benjamins.

2 Omitimos el hecho de que por cotejo de rasgo de caso Nominativo (o de un rasgo PPE) el SUJ se ensambla internamente en la posición superior de ESP de T. 
Arad, MaYA. 1998. VP Structure and the Syntax-Lexicon Interface. Tesis doctoral, UCL. MIT Occasional Papers in Linguistics 16. Cambridge, MA: MIT.

1999. On "little v". En K. Arregi, B. Bruening, C. Krause y V. Lin (eds.). Papers on Morphology and Syntax: Cycle One, MIT Working Papers in Linguistics 33, pp. 1-25. Cambridge, MA: MIT.

Berkes, Eva. 2001. Aproximación minimalista a las categorías funcionales del ámbito oracional: la derivación por fase. Tesis doctoral, Universidad Complutense de Madrid.

CARSTON, Robyn. 1998. The Semantics/pragmatics distinction: a view from Relevance Theory. UCL Working Papers in Linguistics 10: 303-329. 44.

Chомsкy, Noam. 1981. Lectures on Government and Binding. Dordrecht: Foris. 1995. The Minimalist Program. Cambridge, MA: MIT Press. 1998. Minimalist Inquiries: The Framework. MIT Occasional Papers in Linguistis, $\mathrm{N}^{\mathrm{er}}$ 15, Cambridge: MIT Press. 2005. Three factors in Language Design. Cambridge, MA: MIT Press.

Escandell Vidal, María Victoria y Manuel Leonetti. 1997. Categorías funcionales y semántica procedimental. En Actas del Congreso Internacional de Gramática, Universidad de la Laguna, Madrid, Ed. Clásicas, Vol. I, pp. 363-378.

Grice, H. PAul. 1991 [1975]. Lógica y conversación. En Luis Manuel Valdés Villanueva (comp.). La búsqueda del significado. Madrid: Tecnos.

Hale, Ken y Samuel J. Keyser. 1993. On Argument Structure and the Lexical Expression of Syntactic Relations. En Ken Hale y Samuel J. Keyser (eds.). The View from Building 20: Essays in Honour of Sylvian Bromberger, pp. 53-109. Cambridge, MA: MIT Press.

Kayne, Richard. 1994. The Antisymmetry of Syntax. Cambridge, MA: MIT Press.

Leonetti, Manuel. 2001. Gramática y Teoría de la Relevancia (manuscrito). UNR. 2004. Specificity and Differential Object Marking in Spanish. Catalan Journal of Linguistics 3: 75-114.

Pollock, Jean-Yves. 1989. Verb Movement, Universal Grammar, and the Structure of IP. Linguistic Inquiry 20/3: 365-424.

Sperber, Dan y Deirdre Wilson. 1995 [1986]. Relevance: Communication and Cognition. Oxford: Blackwell y Cambridge, MA: Harvard University Press. 